\title{
The Successful Use of Extracorporeal Membrane Oxygenation in Systemic Lupus Erythematosus-Induced Diffuse Alveolar Haemorrhage
}

\author{
Faye Pais, Mohamed Fayed, Timothy Evans \\ Division of Pulmonary Critical Care Medicine, University of California, San Francisco - Fresno, Fresno, California, USA
}

\section{Doi: 10.12890/2016_000515- European Journal of Case Reports in Internal Medicine - @ EFIM 2016}

\begin{abstract}
Received: 08/10/2016
Accepted: $15 / 10 / 2016$

Published: $14 / 11 / 2016$

How to cite this article: Pais F, Fayed M, Evans T. The successful use of extracorporeal membrae oxygenation in systemic lupus erythematosus-induced diffuse alvelolar haemorrage. EJCRIM 2016;3: doi:10.12890/2016_000515.
\end{abstract}

Conflicts of Interests: The Authors declare that there are no competing interests.

This article is licensed under a Commons Attribution Non-Commercial 4.0 License

\section{ABSTRACT}

Diffuse alveolar haemorrhage (DAH) is a catastrophic pulmonary complication of systemic lupus erythematosus. It can result in refractory hypoxaemia despite mechanical ventilation. Increasing lung compliance and worsening pulmonary hypertension can potentiate cardiogenic shock from acute right ventricular failure. In such patients with cardiopulmonary collapse, veno-arterial (V-A) ECMO maybe a viable option that can provide the required haemodynamic support. However, the use of V-A ECMO in such patients is limited due to an associated increased risk of bleeding. Our case report describes the successful use of V-A ECMO without the use of systemic anticoagulation in a patient with DAH. Despite the absence of systemic anticoagulation, no thrombotic complications within the circuit were noted.

\section{LEARNING POINTS}

- Diffuse alveolar haemorrhage (DAH) complicating systemic lupus erythematous can present dramatically with sudden cardiopulmonary collapse.

- Extracorporeal membrane oxygenation (ECMO) can provide temporary haemodynamic support until conventional medical therapy can take effect.

- Despite the historical increased bleeding risk associated with ECMO, it can be safely used in patients with DAH to provide time for underlying organ recovery.

\section{KEYWORDS}

Alveolar hemorrhage; extra corporeal membrane oxygenation; lupus

\section{INTRODUCTION}

Diffuse alveolar haemorrhage (DAH) is a rare but catastrophic complication of many systemic diseases. It classically presents with haemoptysis, dropping haemoglobin levels and new radiographic infiltrates. DAH can progress to hypoxaemic respiratory failure requiring mechanical ventilation, which is a poor prognostic marker ${ }^{[1]}$. DAH is seen in systemic lupus erythematosus (SLE), although more commonly in patients with underlying lupus nephritis ${ }^{[1]}$. Despite optimal medical therapy, outcomes remain highly variable. Extracorporeal membrane oxygenation (ECMO) has been used as a bridge or temporary support in potentially severe, reversible pulmonary and/or cardiac dysfunction refractory to conventional medical therapy. However, the use of systemic anticoagulation while on ECMO may be associated with increased risk of bleeding ${ }^{[2]}$ that could limit its utility in patients with DAH. In this clinical vignette, we describe the successful use of veno-arterial 
extracorporeal membrane oxygenation (V-A ECMO) without anticoagulation in a patient with refractory hypoxaemia and cardiopulmonary collapse resulting from SLE-induced DAH.

\section{CASE REPORT}

A 38-year-old woman presented with fever, cough, haemoptysis and chest pain for 1 day. She had been diagnosed with SLE-induced type IV lupus nephritis (mixed membranous and diffuse proliferative lupus glomerulonephritis with $45 \%$ crescents) and was being treated with prednisolone and mycophenolate.

On physical examination, her respiratory rate was 25 breaths per minute, pulse 100 beats per minute, blood pressure $122 / 79 \mathrm{mmHg}$ and temperature $38.5^{\circ} \mathrm{C}$ with crackles over the lower lung fields. Initial laboratory test results revealed a haemoglobin of $6.6 \mathrm{~g} / \mathrm{dl}$ and the chest $\mathrm{x}$-ray (Fig. 1A) was consistent with bilateral alveolar infiltrates. An arterial blood gas (ABG) revealed a pH of 7.37, a $\mathrm{PacO}_{2}$ of 26 , a $\mathrm{PaO}{ }_{2}$ of 95 and an $\mathrm{HCO}_{3}$ of 15 on an $\mathrm{FIO}_{2}$ of 0.35 on a Venturi mask. The patient was diagnosed with $\mathrm{DAH}$ and immediately started on high-dose methyl prednisolone and mycophenolate (at her home dose).

Over the next 2 hours, she continued to have haemoptysis and worsening hypoxaemia with increasing bilateral infiltrates on CT scan (Fig. 1B). She was intubated and transferred to the intensive care unit (ICU) where she had a pulseless electrical arrest but was revived with chest compressions. A bedside echocardiogram demonstrated a hypokinetic and dilated right ventricle with markedly elevated pulmonary artery pressure for which she was started on dobutamine.

Despite maximal ventilatory management, sedation, neuro-muscular blockade and maximal vasopressor support, the patient did not improve. She had worsening respiratory acidosis on $\mathrm{ABG}\left(\mathrm{pH}\right.$ of 6.96, a $\mathrm{PaCO}_{2}$ of 61 , a $\mathrm{PaO}_{2}$ of 59 and an $\mathrm{HCO}_{3}$ of 14 on an $\mathrm{FIO}{ }_{2}$ of 1.0 ) and hence $V-A$ ECMO was initiated

A chest x-ray (Fig. 1C) obtained the following day showed worsening airspace disease. However, the patient was continued on $\mathrm{V}$-A ECMO. The ventilator was maintained at an $\mathrm{FIO}_{2}$ of 0.5 , positive end-expiratory pressure (PEEP) of 8 , tidal volume of $185 \mathrm{ml}$ and respiratory rate of 8 breaths per minute. Intravenous methyl prednisolone and plasmapheresis were continued.

On day 3 of V-A ECMO, the patient demonstrated improved cardiac output, decreased vasopressor requirement and increased lung compliance. Her chest x-ray (Fig. 1D) and ABG also showed significant improvement ( $\mathrm{pH}$ of 7.48, a Paco of $_{3}$, a $\mathrm{PaO}_{2}$ of 267 and an $\mathrm{HCO}_{3}$ of 27 on an $\mathrm{FIO}_{2}$ of 0.5 and PEEP of 8). V-A ECMO was discontinued on day 3. The patient was successfully extubated on day 5 and plasmapheresis was stopped on day 6. Over the next 3 weeks she improved steadily and was finally discharged home.

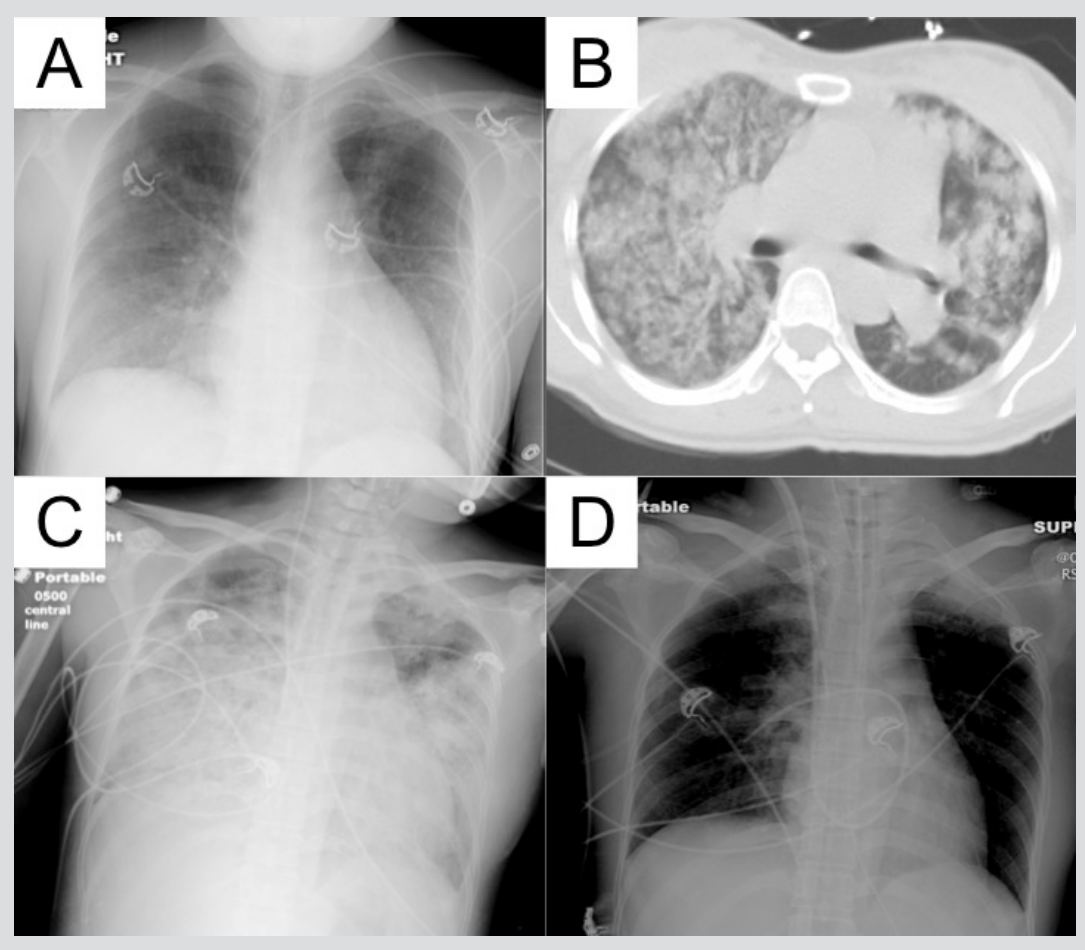

Figure 1. Patient's imaging (A) at admission, (B) 2 hours after admission, (C) 11 hours after the initiation of veno-arterial extracorporeal membrane oxygenation ( $V-A$ ECMO), and (D) on the third day of $V-A$ ECMO. 


\section{DISCUSSION}

Diffuse alveolar haemorrhage (DAH) is characterized by the extravasation of red blood cells into the pulmonary alveolar spaces from a disruption of the alveolar capillary interface. Histologically, DAH can arise from small vessel vasculitis (capillaritis), diffuse alveolar damage or bland haemorrhage from underlying coagulopathy and/or pulmonary venous hypertension ${ }^{[3]}$. All these histological sub-types can be associated with SLE-induced DAH ${ }^{[1,3]}$, although pulmonary capillaritis is the most common. The treatment for SLE-induced DAH includes high-dose corticosteroids often in combination with cyclophosphamide, mycophenolate or rituximab. Despite the lack of controlled clinical trials, plasmapheresis is often used in the acute setting.

Our patient had SLE-induced DAH that resulted in fulminant hypoxaemic respiratory failure, coupled with refractory obstructive shock. Since its first successful use in 1972, veno-venous (V-V) ECMO has gained popularity as a salvage therapy in severe but potentially reversible hypoxaemic respiratory failure. However, V-A ECMO maybe a necessary alternative in the setting of acute right heart failure from severe acute lung injury. Traditionally, the use of systemic anticoagulation while on ECMO has limited its use in patients with increased risk of bleeding ${ }^{[2]}$. However, recent technical advances in the extracorporeal circuit have allowed for the reduction ${ }^{[4]}$ or omission of systemic anticoagulation in this sub-group of patients $\mathrm{S}^{[5]}$. Here we describe the successful use of V-A ECMO in our patient who had massive haemoptysis with hypoxaemic respiratory failure from DAH coupled with severe acute right heart failure from severely elevated pulmonary pressures. In this advanced but potentially reversible setting, ECMO provided our patient with temporary haemodynamic support. As evidenced by our patient's remarkable recovery during this period, it can be speculated that V-A ECMO may have provided our patient with the additional time required for the recovery of the with the help of appropriate medical management.

Our case report contributes to the scant literature supporting the use of V-A ECMO as a viable option in patients with refractory hypoxaemia and reversible haemodynamic collapse from acute right heart failure due to acute lung injury.

\section{REFERENCES}

1. Zamora MR, Warner ML, Tuder R, Schwarz MI. Diffuse alveolar hemorrhage and systemic lupus erythematosus: clinical presentation, histology, survival, and outcome. Medicine 1997;76:192-202.

2. Extracorporeal Life Support Organization. ELSO guidelines for cardiopulmonary extracorporeal life support. Version 1.3. Ann Arbor, MI; 2013.

3. Lara AR, Schwarz MI. Diffuse alveolar hemorrhage. Chest 2010;137:1164-1171.

4. Abrams D, Agerstrand CL, Biscotti M, Burkart KM, Bacchetta M, Brodie D. Extracorporeal membrane oxygenation in the management of diffuse alveolar hemorrhage. ASAIO J 2015;61:216-218.

5. Muellenbach RM, Kredel M, Kunze E, Kranke P, Kuestermann J, Brack A, et al. Prolonged heparin-free extracorporeal membrane oxygenation in multiple injured acute respiratory distress syndrome patients with traumatic brain injury. J Trauma Acute Care Surg 2012;72:1444-1447. 